\title{
Disorders Involving a Persistent Craniopharyngeal Canal: A Case Series
}

\author{
Seerat K. Poonia ${ }^{1}$ Diego Cazzador ${ }^{2}$ Adam C. Kaufman ${ }^{1}$ Michael A. Kohanski ${ }^{1}$ \\ Edward C. Kuan ${ }^{30}$ Charles C. L. Tong ${ }^{1}$ Roy D. Carlson ${ }^{1}$ Daniele Borsetto ${ }^{2}$ \\ Enzo Emanuelli ${ }^{2}$ James N. Palmer ${ }^{1}$ Nithin D. Adappa ${ }^{1}$
}

${ }^{1}$ Department of Otorhinolaryngology - Head and Neck Surgery,

Address for correspondence Nithin D. Adappa, MD, Department of Perelman School of Medicine, University of Pennsylvania,

Philadelphia, Pennsylvania, United States

2 Otorhinolaryngology Unit, Department of Neurosciences, University

of Padua, Padua, Italy

${ }^{3}$ Department of Otolaryngology - Head and Neck Surgery, University of Otorhinolaryngology - Head and Neck Surgery, Perelman School of Medicine, University of Pennsylvania, 3400 Spruce Street, 5th Floor Silverstein Building, Philadelphia, PA 19104, United States

California, Irvine School of Medicine, Orange, California, United States

(e-mail: Nithin.Adappa@uphs.upenn.edu).

J Neurol Surg B 2020;81:562-566.

\begin{abstract}
Objectives A persistent craniopharyngeal canal (CPC) is a rare embryologic remnant that presents as a well-corticated defect of the midline sphenoid body extending from the sellar floor to the nasopharynx. Our case series aims to describe three unique presentations of this congenital anomaly and their subsequent management.

Design Retrospective review.

Setting Tertiary academic medical center.

Participants Patients who underwent endoscopic transnasal surgical repair of a CPC lesion.

Main Outcome Measures Resolution of symptoms and surgical outcomes.

Results A total of three patients were identified. The clinical presentation varied, however, all cases prompted further imaging which demonstrated a persistent CPC and associated pathologic lesion. The presentation of a persistent CPC with nasal obstruction and subsequent iatrogenic cerebrospinal fluid leak as in Case 1 demonstrates the

Keywords

- craniopharyngeal canal

- congenital

- endoscopic

- skull base

- surgery

- cerebrospinal fluid importance of imaging in this work-up. Cases 2 and 3 in the series were representative of the larger subset of patients in the literature who present with the defect incidentally but still warrant surgical management. Nonetheless, a standard approach to diagnosis with preoperative imaging and subsequent transnasal endoscopic repair of the skull base defect was undertaken.

Conclusion The persistent CPC is a rare congenital anomaly associated with diverse pathology and careful review of preoperative radiology is critical to the management. When warranted, subsequent surgical repair and reconstruction is associated with excellent postoperative outcomes.
\end{abstract}

received

November 8, 2018

accepted

May 11, 2019

published online

June 21, 2019 (c) 2020 Georg Thieme Verlag KG

Stuttgart · New York
Dol https://doi.org/

10.1055/s-0039-1692638.

ISSN 2193-6331. 


\section{Introduction}

A persistent craniopharyngeal canal (CPC) is a rare embryologic remnant that presents as a well-corticated defect of the midline sphenoid body extending from the sellar floor to the nasopharynx. It is thought that the structure either results from incomplete closure of Rathke's pouch or is a former vascular channel remaining from ossification of the sphenoid during development. ${ }^{1,2}$ CPCs of variable sizes and with a range of associated lesions have been described in the literature, but the rarity of presentation blunts appreciation of their clinical presentation and significance in practice.

In a published case series, Abele et al $^{1}$ classified CPCs into three types according to size and contents based on radiologic appearance. Small incidental CPCs are termed type 1, and in this case series, all nine patients presented with symptoms unrelated to their defect, or associated with preexisting congenital anomalies. Medium-sized canals containing an ectopic or inferiorly displaced adenohypophysis are classified as type $2 \mathrm{CPCs}$, presenting with pituitary dysfunction, headache, coloboma, and hydrocephalus in seven patients. Large canals with associated encephaloceles are type $3 \mathrm{~A}$, presenting with growth hormone deficiency, generalized hypopituitarism, diabetes insipidus, or meningitis in four patients. Finally, CPCs with tumors are type 3B and canals with both are type $3 C$. Of the remaining nine types 3B and 3 C CPCs, patients presented with a nasopharyngeal mass, growth hormone deficiency, duplicated pituitary gland, or were incidental findings on unrelated imaging.

Correct diagnosis of a CPC plays a significant role in further work-up of the lesion related to pituitary dysfunction, investigating any associated abnormalities, and with subsequent surgical management. Thus, though uncommon, they are an important entity to consider in the evaluation of nasopharyngeal or midline skull base lesions. Our report describes three pathologic presentations of this rare congenital midline defect and their subsequent management.

\section{Case Presentations}

\section{Case 1}

A healthy 56-year-old woman presented to an outside otolaryngologist with nasal obstruction and was found to have a mass emanating from her nasopharynx. A biopsy of the mass leads to copious drainage of clear fluid that was positive for $\beta-2$ transferrin, concerning for cerebrospinal fluid (CSF). She soon afterward developed meningitic symptoms necessitating transfer to a tertiary academic medical center for further work-up. Of note, as an infant she had developed meningitis of unclear etiology.

After the initiation of broad-spectrum antibiotics, bedrest and stool softeners, computed tomography scan, and magnetic resonance imaging (MRI) were obtained which showed, originating from the pituitary gland, a remnant craniopharyngeal pouch traversing the mid-portion of the sphenoid sinus and extending into the nasopharynx (-Figs. 1 and 2 ).

At this point, the patient was consented for endoscopic transnasal surgical repair. Upon exposing the sphenoid sinus

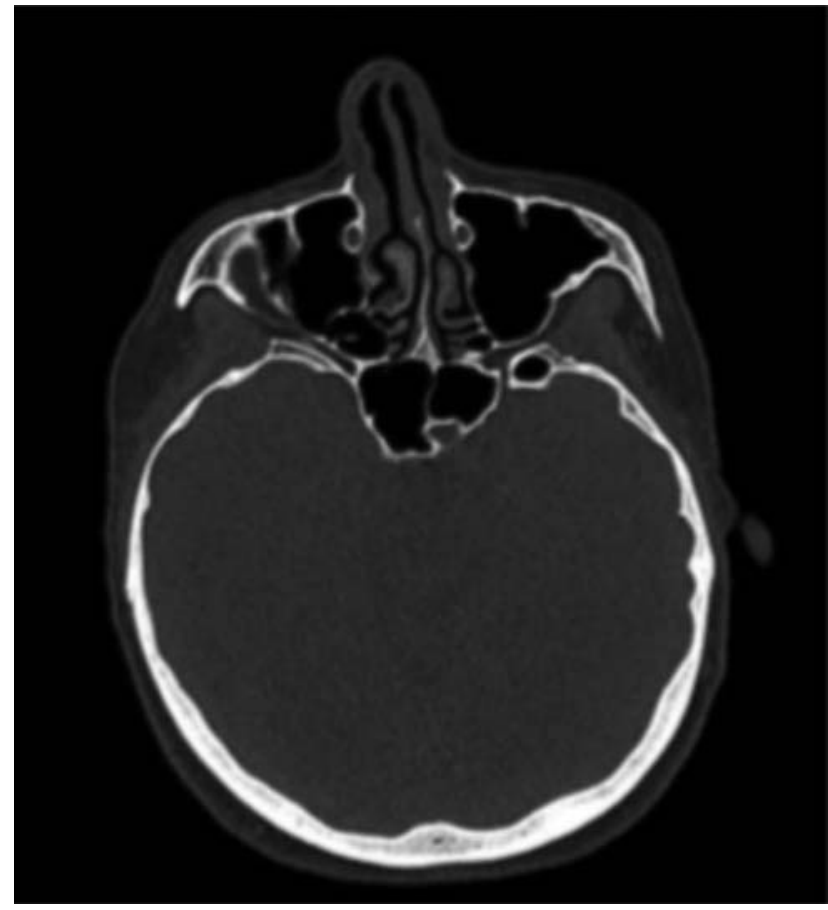

Fig. 1 Axial cut computed tomography scan without contrast demonstrating the start of a dural opening entering the sphenoid sinus just to the left of the intersinus septum.

and sella, the lesion was found to be encased by a bony covering that was removed using a high-speed drill. A dural component was identified within the bony canal which was actively leaking CSF into the sphenoid sinus (-Fig. $\mathbf{3}$ ). The lesion was then resected taking care to avoid damage to the

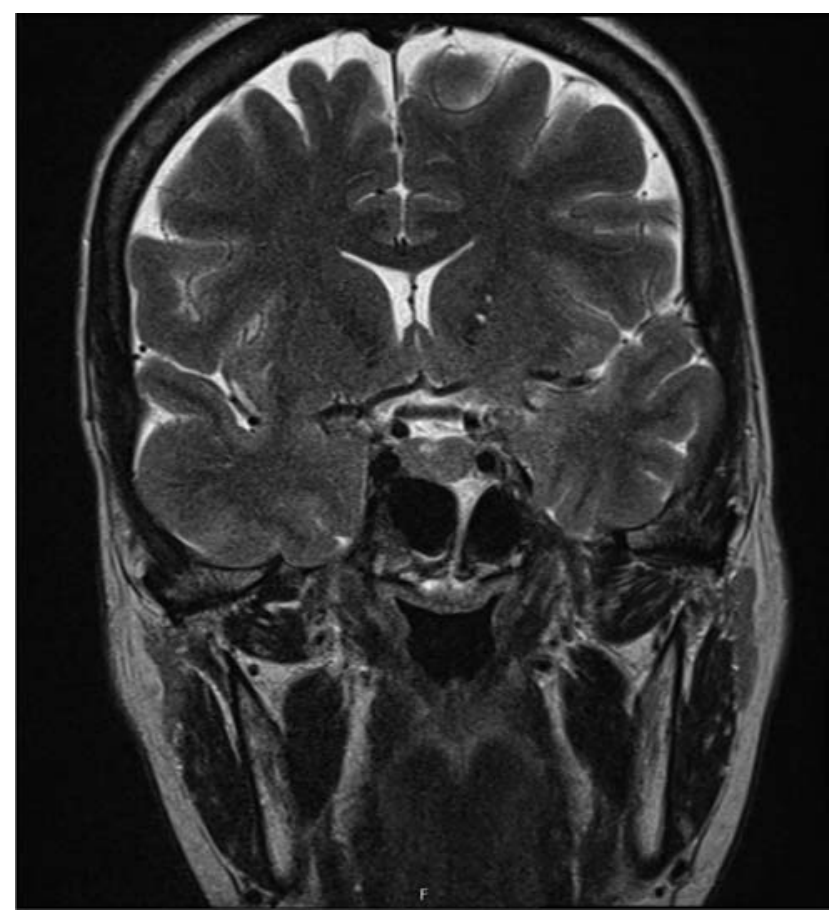

Fig. 2 Coronal cut T2 magnetic resonance imaging which demonstrates a cerebrospinal fluid pocket within a bony canal from the left sella floor inferiorly in the intersinus septum, consistent with a persistent craniopharyngeal canal. 


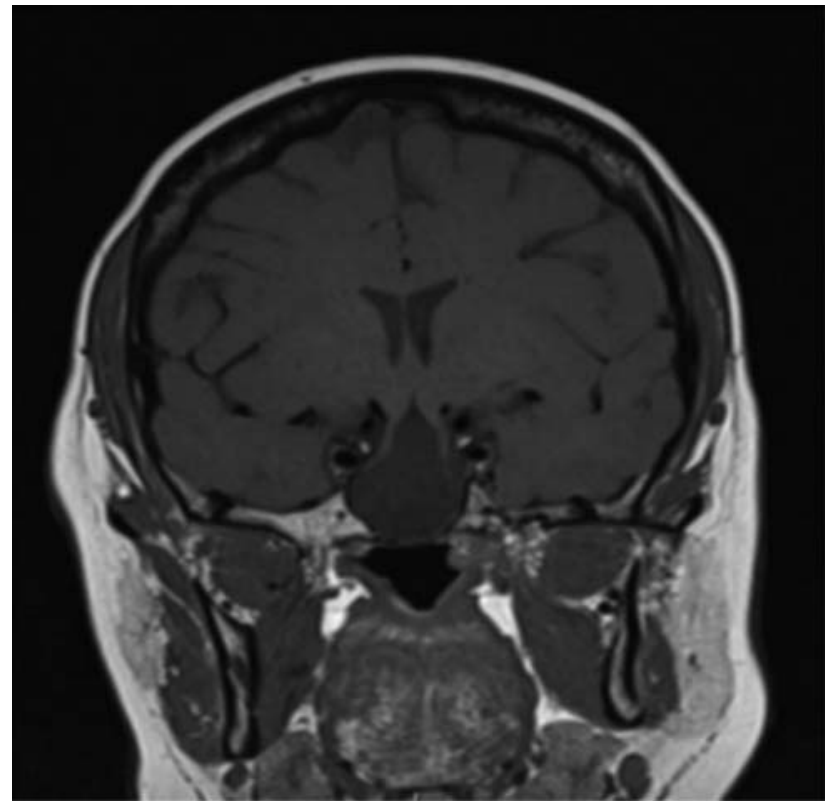

Fig. 3 Coronal T1 magnetic resonance imaging showing a large hypointense lesion in the sphenoid sinus consistent with a meningoencephalocele.

pituitary gland. The CSF leak was repaired with a dural graft matrix underlay and a pedicled nasoseptal flap. She did not develop any further clear fluid rhinorrhea or hypopituitarism subsequent to the surgery.

\section{Case 2}

While investigating the etiology of menstrual irregularities, a massive midline encephalocele was incidentally found on the MRI of a 41-year-old woman ( - Fig. 4). The encephalocele was seen descending through a persistent CPC terminating as a mass in the nasopharynx (-Fig. 5).

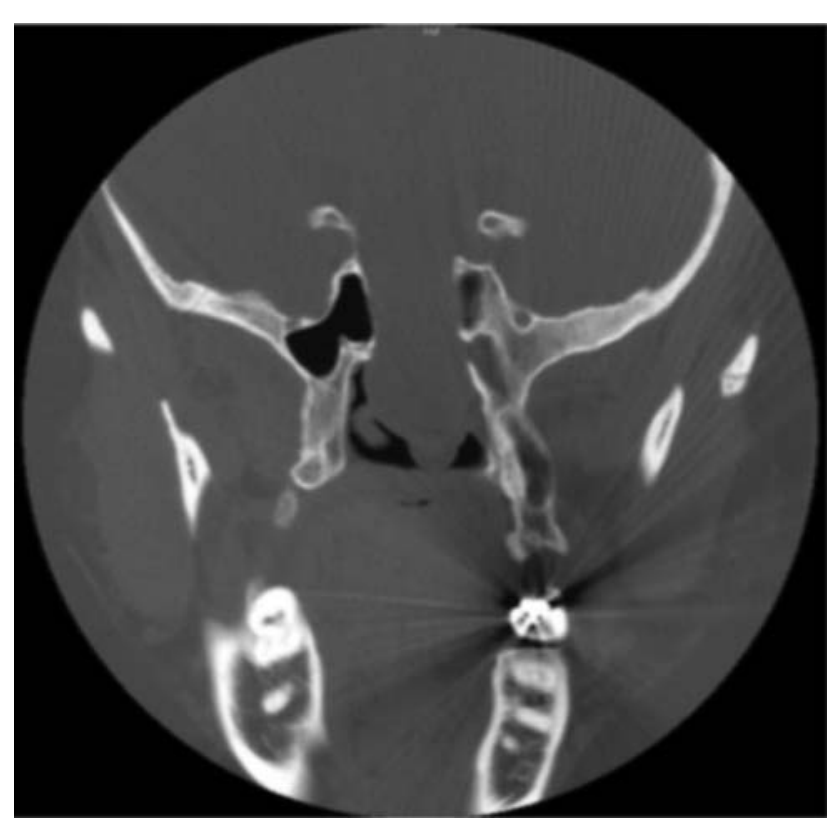

Fig. 4 Coronal computed tomography scan showing a large encephalocele herniating through a midline defect in the sphenoid bone.

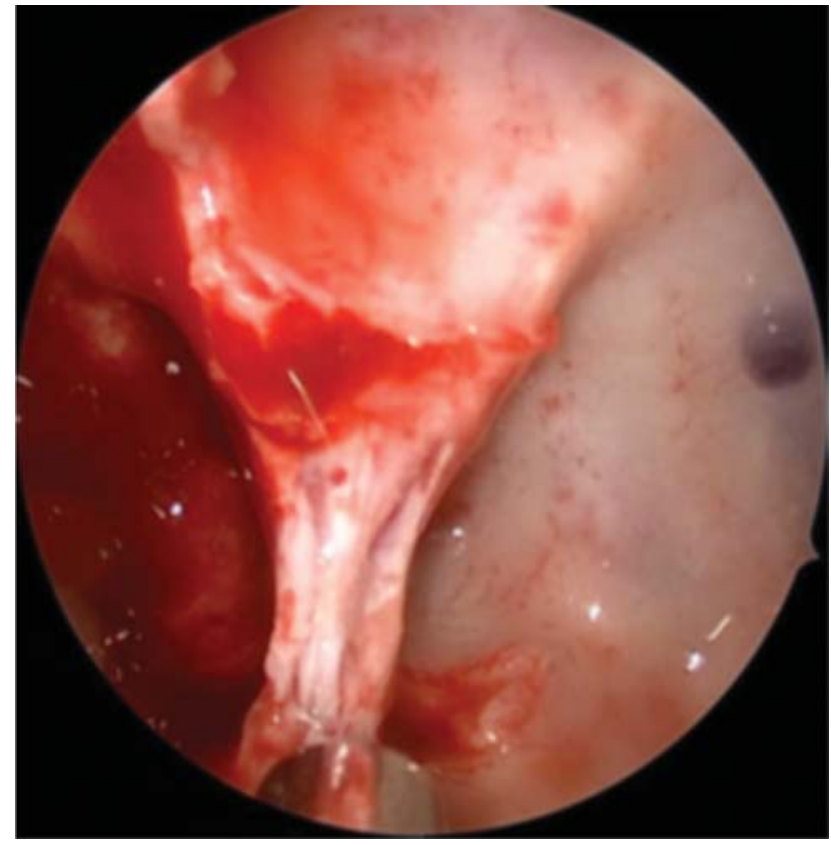

Fig. 5 Intraoperative endoscopic view of a persistent craniopharyngeal canal running within the sphenoid intersinus septum.

She was referred to the rhinology clinic and consented for transseptal surgical excision of the midline skull base lesion. With the sella exposed, the encephalocele was resected and the skull base defect was repaired with a dural graft matrix underlay, septal bone, and a middle turbinate free mucosal graft. She recovered without issues, and there was no evidence of ectopic pituitary tissue on follow-up pathology. Additionally, she did not develop any visual disturbances or hypopituitarism.

\section{Case 3}

A 14-year-old girl with macrocephaly, cerebral aqueduct stenosis, and consequent hydrocephalus who had undergone a previous ventriculoperitoneal shunt placement at the age of 1 month presented to the clinic with a history of recurrent meningitis. On MRI, she was found to have severe Chiari malformation type 2 and a persistent CPC. Although noted to have a small pituitary gland, she had normal pituitary function on further work-up.

Given her history of recurrent meningitis, she was brought to the operating room for evaluation and reconstruction of the CPC defect. The inferior portion of the canal was isolated after drilling the rostrum. The skull base defect was repaired with a gasket seal technique. The inferior portion of the canal was sealed with an underlay of fascia lata, a bone graft from the middle turbinate, another piece of fascia lata as an overlay, and a middle turbinate free mucosal graft. She has developed no subsequent CSF leak or meningitis on 6 years follow-up.

\section{Discussion}

The persistent CPC is a rare congenital skull base defect extending from the floor of the sella turcica inferiorly to the 
nasopharynx. It is thought to be related to the route of ascent of Rathke's pouch, the precursor of the adenohypophysis, to the sella turcica. Others believe it to be a remnant of a former vascular channel which develops during ossification of the sphenoid bone, a theory first advocated by Arey. ${ }^{2}$ In either case, its presence is associated with diverse pathology which presents in varied manners, and if warranted surgical management is associated with excellent outcomes when aided by thorough clinical and radiological evaluations.

This anomaly is known to be present during childhood with a prevalence of up to $10 \%$ in two studies of infant anatomic specimens, but is often first diagnosed in older age groups. $^{1-3}$ In two reports, infants presented with postnatal dyspnea and cyanosis secondary to a meningoencephalocele associated with a CPC causing nasopharyngeal obstruction. In both cases radiological assessment led to the appropriate diagnosis and resolution with surgical treatment. ${ }^{4-6}$ Although in infants, these reports taken together with Case 1 highlight the unique presentation of meningoencephaloceles with nasal obstruction. They demonstrate the importance of considering this rare entity prior to proceeding with any interventions that may cause iatrogenic CSF leak or hypopituitarism. On the contrary, spontaneous CSF leak is exceedingly rare. In the largest case series published to date of 29 CPCs, no patients presented with a CSF leak. ${ }^{1}$

Furthermore, Currarino et al described a close relationship of large CPCs with transsphenoidal meningoencephaloceles often presenting as a nasopharyngeal mass, although not always. In our case series of three meningoencephaloceles, one patient presented with nasal obstruction related to a presumed nasopharyngeal mass, with the others presenting incidentally. The Currarino et al's study also found these lesions to co-occur frequently with craniofacial anomalies including hypertelorism, midfacial cleft, cleft lip/palate, abnormal optic tracts, and orbital malformations and suggested early on that patients with the defect are prone to herniation of the third ventricle and the pituitary gland. ${ }^{7}$ More focused reports have demonstrated the presence of the adenohypophysis in the persistent CPC, and likewise lesions which originate there including hamartomas ${ }^{8}$ and adenomas. ${ }^{9}$ Another case study reported two patients who presented with hypopituitarism following a nasal biopsy of an ectopic adenohypophysis which was presumed to be polyposis. ${ }^{10}$ Chen et al described an exceedingly rare case of an infrasellar craniopharyngioma which arose along a CPC. In all instances, the relationship of the lesion with a persistent CPC was delineated only on obtaining appropriate imaging. ${ }^{11}$ The importance of this concept is clearly demonstrated in our review of Case 1 where biopsy was performed prior to any imaging and resulted in an iatrogenic CSF leak and subsequent meningitis. However, in Cases 2 and 3, preoperative imaging was performed, and thus appropriate diagnosis and classification of the lesions as type 3A CPCs could be undertaken. This ability informed the subsequent management of these patients.

As discussed, an approach to classify CPCs into three types based on radiologic appearance has been described by Abele et al and is relevant in discussing subsequent management. ${ }^{1}$
In our series, all three cases would be classified as type $3 \mathrm{~A}$ CPCs based on radiography demonstrating the presence of a meningoencephalocele. It is important however to recognize that this description alone does not allow for adequate determination of the treatment plan. Although radiology is critical in diagnosis of this entity, it should not be relied on alone for subsequent management and discussion of surgical intervention should be undertaken on a case-by-case basis. Appropriate and safe assessment of CPCs requires thorough and stepwise management of each patient's clinical presentation and comorbidities, as well as elucidation of any other complex associations. In Case 1, the patient may not have warranted surgery at her initial presentation, but she did require intervention given her persistent CSF leak despite conservative management. Cases 2 and 3 are representative of a large subset of patients who present incidentally during work-up of symptoms unrelated to the CPC or in association with other congenital malformations, respectively, but that warranted surgical intervention given the extent of the lesions.

\section{Conclusion}

The persistent CPC is a rare midline congenital defect and may present differently based on the size and contents of the encephalocele or tumor if present. Thus, careful review of preoperative radiology is critical to the management. Nonetheless, subsequent surgical repair and reconstruction is associated with excellent postoperative outcomes.

\section{Note}

This article was presented as a poster presentation at the 2018 North American Skull Base Society Annual Meeting.

\section{Funding}

There are no sources of financial support to disclose.

Conflict of Interest

None.

\section{References}

1 Abele TA, Salzman KL, Harnsberger HR, Glastonbury CM. Craniopharyngeal canal and its spectrum of pathology. AJNR Am J Neuroradiol 2014;35(04):772-777

2 Arey LB. The craniopharyngeal canal reviewed and reinterpreted. Anat Rec 1950;106(01):1-16

3 Kaushik C, Ramakrishnaiah R, Angtuaco EJ. Ectopic pituitary adenoma in persistent craniopharyngeal canal: case report and literature review. J Comput Assist Tomogr 2010;34(04):612-614

4 Richter E, Pirsig W, Tänzer A. A persistent craniopharyngeal canal combined with a meningoencephalocele (author's transl) [in German]. HNO 1975;23(08):240-245

5 Braun M, Boman F, Hascoet JM, Chastagner P, Brunet A, Simon C. Brain tissue heterotopia in the nasopharynx. Contribution of MRI to assessment of extension. J Neuroradiol 1992;19(01):68-74

6 Akyel NG, Alımlı AG, Demirkan TH, Sivri M. Persistent craniopharyngeal canal, bilateral microphthalmia with colobomatous cysts, ectopic adenohypophysis with Rathke cleft cyst, and ectopic neurohypophysis: case report and review of the literature. Childs Nerv Syst 2018;34(07):1407-1410 
7 Currarino G, Maravilla KR, Salyer KE. Transsphenoidal canal (large craniopharyngeal canal) and its pathologic implications. AJNR Am J Neuroradiol 1985;6(01):39-43

8 Kizilkilic O, Yalcin O, Yildirim T, Sener L, Parmaksiz G, Erdogan B. Hypothalamic hamartoma associated with a craniopharyngeal canal. AJNR Am J Neuroradiol 2005;26(01):65-67

9 Ekinci G, Kiliç T, Baltacioğlu F, et al. Transsphenoidal (large craniopharyngeal) canal associated with a normally functioning pituitary gland and nasopharyngeal extension, hyperprolactinemia, and hypothalamic hamartoma. AJR Am J Roentgenol 2003; 180(01):76-77

10 Hughes ML, Carty AT, White FE. Persistent hypophyseal (craniopharyngeal) canal. Br J Radiol 1999;72(854):204-206

11 Chen CJ. Suprasellar and infrasellar craniopharyngioma with a persistent craniopharyngeal canal: case report and review of the literature. Neuroradiology 2001;43(09):760-762 